\title{
Toxoplasma gondii Infection in Dustmen in Northeastern China: A Case-Control Seroprevalence Study
}

\author{
Ruo-Lan Jiang,, Quan Zhao $\left({ }^{1},{ }^{2}\right.$ Jing Jiang, ${ }^{2}$ Xue-Long Chen, \\ Xiao-Xuan Zhang $\left(\mathbb{0},{ }^{3}\right.$ and Xiang $\mathrm{Wu}\left(\mathbb{1}^{1}\right.$ \\ ${ }^{1}$ Department of Parasitology, Xiangya School of Medicine, Central South University, Changsha, Hunan Province 410013, China \\ ${ }^{2}$ College of Animal Science and Technology, Changchun Sci-Tech University, Shuangyang, Jilin Province 130600, China \\ ${ }^{3}$ College of Animal Science and Veterinary Medicine, Heilongjiang Bayi Agricultural University, Daqing, \\ Heilongjiang Province 163319, China
}

Correspondence should be addressed to Quan Zhao; zhaoquan0825@163.com and Xiang Wu; wxspring@126.com

Received 19 September 2018; Revised 7 November 2018; Accepted 12 November 2018; Published 19 December 2018

Academic Editor: Valeria Cavalcanti Rolla

Copyright (C) 2018 Ruo-Lan Jiang et al. This is an open access article distributed under the Creative Commons Attribution License, which permits unrestricted use, distribution, and reproduction in any medium, provided the original work is properly cited.

\begin{abstract}
Background. Toxoplasmosis is caused by an intracellular parasite Toxoplasma gondii, which can infect many hosts including humans. Methods. In order to estimate whether dustmen are more susceptible to T. gondii, a case-control study was conducted containing 332 dustmen from Jilin and Heilongjiang in Northeastern China, as well as 332 general populations from the same regions as control subjects. Serum samples were tested IgG and IgM antibodies to T. gondii using the enzyme-linked immunosorbent assay (ELISA). Results. The overall anti-T. gondii IgG was $15.06 \%(50 / 332)$ in dustmen compared with $9.64 \%(32 / 332)$ in the controls $(P=0.0337)$. Also, $5(1.51 \%)$ dustmen had anti-T. gondii IgM antibodies compared with $2(0.60 \%)$ control individuals $(P=0.2543)$. A significant association was only found between dustmen and level of T. gondii IgG in comparison with the control subjects. Seroprevalence of T. gondii IgG antibodies in male dustmen was significant higher than male control subjects $(P=0.0399)$. Dustmen from Jilin had the significant higher T. gondii IgG rate $(P=0.0143)$, in comparison with the control subjects from Jilin. Moreover, dustmen raising cat at home had the significant higher T. gondii IgG rate $(P=0.0097)$, in comparison with the control subjects. Risk factor analysis suggested that raising cat at home and not having habits of washing hand before eating were mainly related to the $T$. gondii infection in dustmen. Conclusions. This is the first record of seroprevalence of T. gondii infection in dustmen in Jilin and Heilongjiang provinces in Northeastern China. These findings also suggest that the government departments should pay close attention to the toxoplasmosis in dustmen in Northeastern China.
\end{abstract}

\section{Introduction}

Toxoplasmosis is a zoonotic disease caused by an intracellular protozoan parasite Toxoplasma gondii, which has a global distribution and wide-ranges of hosts $[1,2]$. Felines are the only definitive hosts for the parasite and virtually all warmblooded animals are intermediate hosts such as humans $[3,4]$. Foodborne and waterborne transmission as well as transplacental infection are the major routes for transmission of $T$. gondii $[5,6]$. Although $1 / 3$ of the world populations are seropositive for T. gondii, most of them are asymptomatic [3]. However, it can cause various severe diseases, and even death in immunocompromised individuals [7].
In view of such severe situations, it is essential to investigate the status of $T$. gondii infection in workers in different professions. There are some investigations focusing on detection of $T$. gondii in people worked at different professions recently. For example, Alvarado-Esquivel et al. demonstrated that $23(12.0 \%)$ of 192 truck drivers were antiT. gondii IgG antibodies positive in Mexico [8]; moreover, a total of 278 out of 464 migrant workers have been detected as Sahimin et al. detected as T. gondii-positive in Malaysia [9]. The similar studies have also been conducted in China, such as involving in livestock and poultry breeding and processing workers [10]. Dustman is an occupation prone to acquire several infections and toxoplasmosis in one of 
them [11]. It is important that information regarding T. gondii infection in dustmen is still scarce [11], especially in China. Therefore, a case-control study was conducted containing 332 dustmen from Jilin and Heilongjiang in Northeastern China, as well as 332 general populations from the same regions as control subjects, to determine the seroprevalence of $T$. gondii in dustmen and to estimate whether dustmen are more susceptible to be T. gondii-infected compared with general populations in Jilin and Heilongjiang in Northeastern China.

\section{Materials and Methods}

2.1. Study Design and Population. The study was approved by the Changchun Sci-Tech University. Participants were made aware of the aim of the study. A total of 664 blood samples (including 332 dustmen and 332 control subjects) were collected from Jilin Province $\left(41^{\circ} \mathrm{N}-46^{\circ} \mathrm{N}, 122^{\circ} \mathrm{E}-131^{\circ} \mathrm{E}\right)$ and Heilongjiang Province $\left(43^{\circ} 26^{\prime} \mathrm{N}-53^{\circ} 33^{\prime} \mathrm{N}, 121^{\circ} 11^{\prime} \mathrm{E}-135^{\circ} 05^{\prime} \mathrm{E}\right)$ in Northeastern China, January 2017 and March 2018. The individuals' occupations and names were not recorded to ensure confidentiality. The purposes and procedures of the study were explained to all participants, and written informed consent was obtained from them all. The sera were collected with agreements from the volunteers. Control sera were collected from volunteers. Information regarding gender, age, whether raising cat at home, whether having habits of washing hands before meals, or geographic region was recorded.

2.2. Sample Collection and Serological Tests. About $5 \mathrm{~mL}$ of venous blood was collected aseptically from each participant in Eppendorf tubes and kept at room temperature for $2 \mathrm{~h}$. Then serum was separated from the whole blood by centrifugation at 3,000 rpm for $10 \mathrm{~min}$, which was labeled and frozen at $-20^{\circ} \mathrm{C}$ until use. Testing for T. gondii serology (IgG and IgM) was performed using commercial enzyme immunoassay kits (Demeditec Diagnostics $\mathrm{GmbH}$, Germany) [4]. Positive, negative, and blank controls were included in every plate. Optical densities were measured by photometer at a wavelength of $450 \mathrm{~nm}$. Values higher than the cut-off $(10 \mathrm{IU} / \mathrm{mL})$ were considered positive.

2.3. Date Analysis. The data of questionnaire covered information such as gender, age, whether raising cat at home, whether having habits of washing hands before meals, or geographic region. The information of questionnaire and experimental results was entered on to an excel spreadsheet and transferred to SPSS v. 19.0 software package (SPSS Inc., USA) [4]. Univariate analysis was used to analyze the association between variables and T. gondii infection. Probability (P) value $<0.05$ was considered as statistically significant in the analysis.

\section{Results and Discussion}

In the present study, a total of 332 dustmen and 332 control individuals were examined (Tables 1 and 2). Of the dustmen, $50(15.06 \%)$ and $5(1.51 \%)$ were detected as anti-T. gondii IgG and IgM antibodies positive, respectively, based on the ELISA methods (Tables 1 and 2). Of them, only two samples were detected as both anti-T. gondii IgG and IgM antibodies positive. The overall prevalence of anti-T. gondii $\operatorname{IgG}$ and IgM antibodies in the control individuals was $9.64 \%$ $(32 / 332)$ and $0.60 \%(2 / 332)$, respectively (Tables 1 and 2$)$. The T. gondii IgG seropositivity rate was $15.70 \%(17 / 147)$ and $16.08 \%(23 / 185)$ in dustmen in Jilin and Heilongjiang, respectively (Table 1 ). There are $8.67 \%$ (13/150) and $10.44 \%$ (19/182) control individuals were examined as T. gondii IgG seropositivity in Jilin and Heilongjiang, respectively (Table 1). The dustmen (15.06\%) had the significant higher T. gondii IgG seroprevalence than control subjects $(9.64 \%, P=0.0337)$ (Table 1); however, no significant difference of T. gondii IgM seroprevalence was found between dustmen group and control group $(P=0.2543)$ (Table 2$)$. In dustmen groups, having cat at home $(P=0.0269)$ and not having habits of washing hand before eating $(P=0.0117)$ were identified to be associated with T. gondii infection in dustmen (Table 1).

T. gondii is one of the most important foodborne zoonotic pathogens, which can cause various severe diseases and even death in immunocompromised individuals [7]. Our case-control study firstly estimated the seroprevalence of T. gondii infection in dustmen. A total of 50 out of 332 dustmen were examined as T. gondii IgG-positive. The overall seroprevalence of anti-T. gondii IgG was $15.06 \%$, which is significant higher than that in control individuals $(9.64 \%$, $32 / 332, P=0.0337)$. However, although the seroprevalence of anti-T. gondii IgM in dustmen was higher than control groups, the difference was not statistically significant $(P$ $=0.2543$ ). These findings suggest that dustmen are more susceptible to be T. gondii-infected than general individuals, and the government departments should pay close attention to the toxoplasmosis in dustmen.

Previous studies have also demonstrated that age was highly related to the $T$. gondii seropositivity [12-15]. Perhaps, elder dustmen had more opportunity to interact with the infective oocysts than younger dustmen. In the present study, seroprevalence of $T$. gondii in dustmen was increase with age (Table 1); however, the difference was not statistically significant $(P=0.6599)$. Moreover, previous studies demonstrated that females were more susceptible to the males, which differ from the present results, revealing that no significant difference in the T. gondii IgG prevalence between males (15.43\%) and females (14.71\%) was found (Table 1), and males had a significant higher T. gondii IgM prevalence than females $(P$ $=0.0210$ ) (Table 2). Also, there was no significant difference statistically among different area of residence (Tables 1 and 2). However, in geographic region groups, dustmen from Jilin had the significant higher T. gondii IgG seroprevalence $(P=0.0143)$ (Table 1$)$ than control subjects from Jilin; the findings suggest that dustmen from Jilin must be must be paid attention to detection of T. gondii infection.

Cat is the only definitive host for $T$. gondii, so they play a crucial role in transmitting T. gondii $[3,16,17]$. Cats discharge feces containing $T$. gondii oocysts into the environment and then become the potential resources of human infection [18]. Therefore, dustmen raising cat at home had a significant higher T. gondii IgG seroprevalence than not raising cat at home groups. Moreover, both T. gondii IgG and IgM 


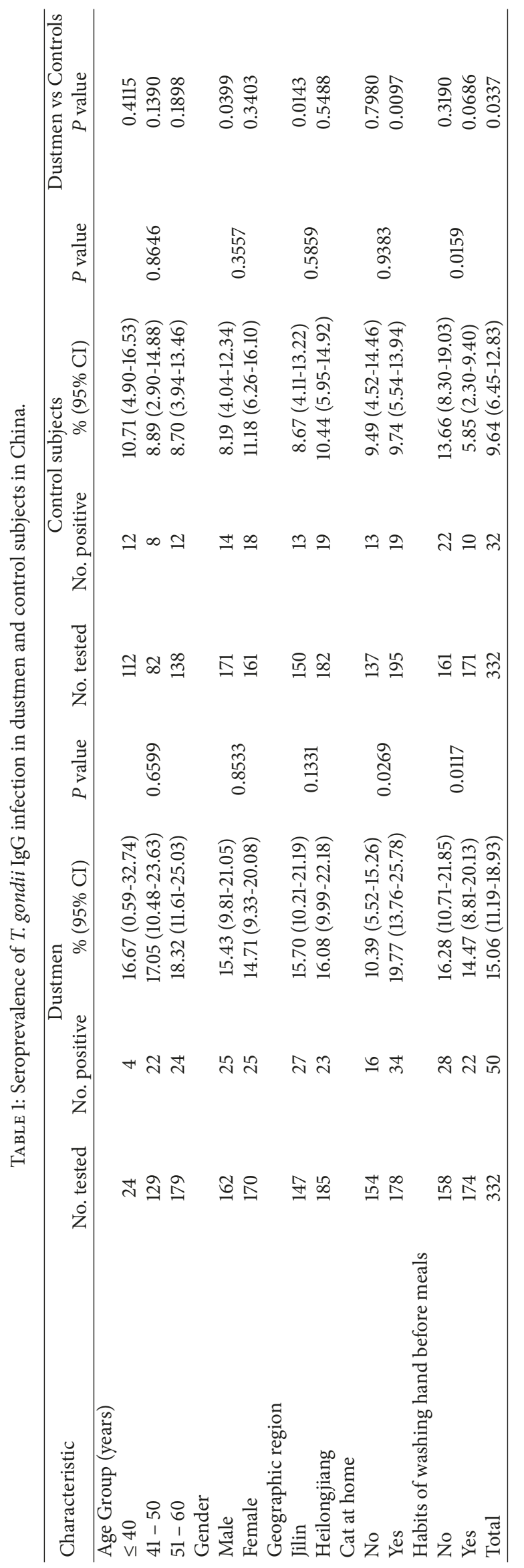




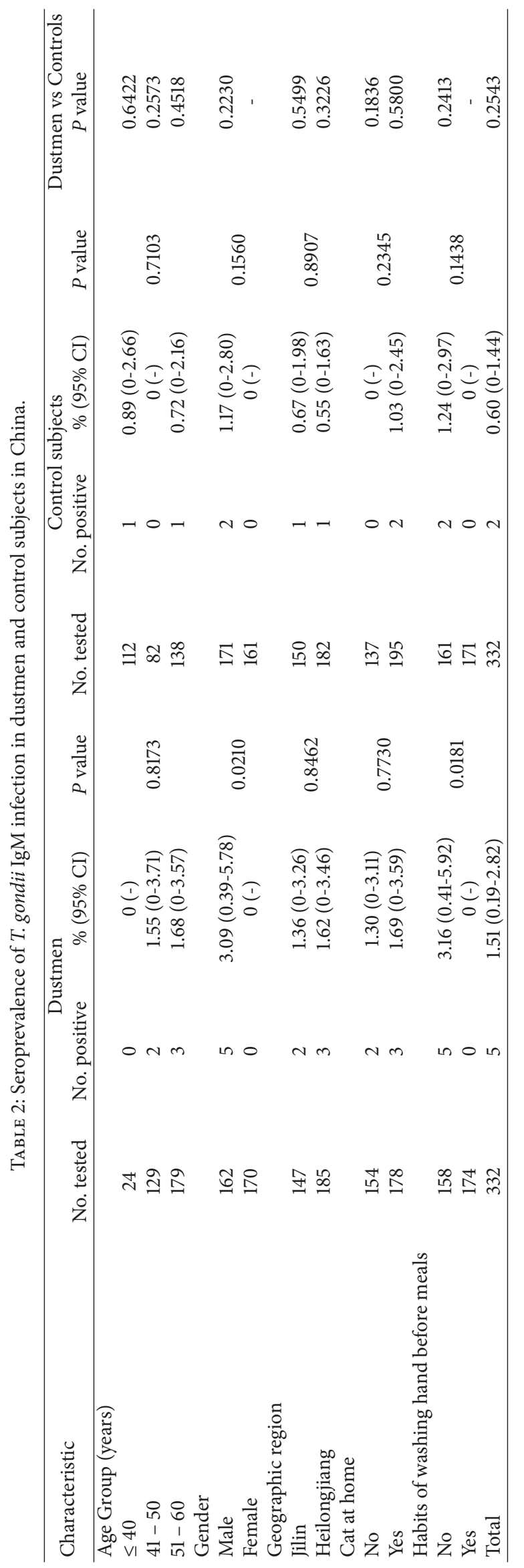


seroprevalence in dustmen who have habits of washing hands before eating were significant lower than those who have not habits of washing hands before eating. The findings suggest the dustmen and other people must cultivate good health habits and wash hands before eating.

\section{Conclusion}

In summary, this is the first report of seroprevalence of T. gondii infection in dustmen in Jilin and Heilongjiang Province in Northeastern China. Dustmen were seen to be more susceptible than control individuals. Seroprevalence of T. gondii infection in dustmen was mainly related to raising cat at home and not washing hands before eating. The findings will provide key and baseline data for prevention and control of toxoplasmosis in dustmen.

\section{Data Availability}

Data on which this article was written could be available as innominate data upon request and approval from Changchun Sci-Tech University.

\section{Conflicts of Interest}

The authors declare that there are no conflicts of interest regarding the publication of this paper.

\section{Acknowledgments}

The authors would like to thank all study participants who took part in the study.

\section{References}

[1] A. Nyambura Njuguna, J. M. Kagira, S. Muturi Karanja, M. Ngotho, L. Mutharia, and N. Wangari Maina, "Prevalence of Toxoplasma gondii and Other Gastrointestinal Parasites in Domestic Cats from Households in Thika Region, Kenya, BioMed Research International, vol. 2017, Article ID 7615810, 6 pages, 2017.

[2] N. Zhou, X. Y. Zhang, Y. X. Li, L. Wang, L. L. Wang, and W. Cong, "Seroprevalence and risk factors of Toxoplasma gondii infection in oral cancer patients in China: a case-control prospective study," Epidemiology and Infection, vol. 13, pp. 1-5, 2018.

[3] X.-X. Zhang, Q. Zhao, C.-W. Shi et al., "Seroprevalence and associated risk factors of Toxoplasma gondii infection in the Korean, Manchu, Mongol and Han ethnic groups in eastern and northeastern China," Epidemiology and Infection, vol. 144, no. 9, pp. 2018-2024, 2016.

[4] R. Jiang, L. Ma, Z. Ma, G. Hou, Q. Zhao, and X. Wu, "Seroprevalence and associated risk factors of Toxoplasma gondii among Manchu pregnant women in northeastern China," Microbial Pathogenesis, vol. 123, pp. 398-401, 2018.

[5] M.-J. Bai, Y. Zou, H. M. Elsheikha et al., "Toxoplasma gondii Infection in Farmed Wild Boars (Sus scrofa) in Three Cities of Northeast China," Foodborne Pathogens and Disease, vol. 14, no. 7, pp. 379-385, 2017.

[6] D. Iddawela, S. M. P. Vithana, and C. Ratnayake, "Seroprevalence of toxoplasmosis and risk factors of Toxoplasma gondii infection among pregnant women in Sri Lanka: A cross sectional study," BMC Public Health, vol. 17, no. 1, p. 930, 2017.

[7] Z. Wang, H. Liu, Z. Ma et al., "Toxoplasma gondii Infection in Immunocompromised Patients: A Systematic Review and Meta-Analysis," Frontiers in Microbiology, vol. 8, Article ID 389, 2017.

[8] C. Alvarado-Esquivel, S. J. Pacheco-Vega, J. Hernández-Tinoco et al., "Toxoplasma gondii infection in interstate truck drivers: a case-control seroprevalence study," Parasites \& Vectors, vol. 8, p. 77, 2015.

[9] N. Sahimin, Y. A. L. Lim, F. Ariffin et al., "Socio-demographic determinants of Toxoplasma gondii seroprevalence in migrant workers of Peninsular Malaysia," Parasites Vectors, vol. 10, no. 1, p. 238, 2017.

[10] Y.-L. Guo, Y.-G. Zou, D.-C. Xu et al., "Analyais of Toxoplasma gondii infection and related factors among special population in Changzhou City, Jiangsu Province," Chinese Journal of Schistosomiasis Control, vol. 28, no. 6, pp. 669-698, 2016.

[11] C. Alvarado-Esquivel, O. Liesenfeld, J. Á. Márquez-Conde et al., "Seroepidemiology of infection with Toxoplasma gondii in waste pickers and waste workers in Durango, Mexico," Zoonoses and Public Health, vol. 55, no. 6, pp. 306-312, 2008.

[12] O. Falusi, A. L. French, E. C. Seaberg et al., "Prevalence and predictors of Toxoplasma seropositivity in women with and at risk for human immunodeficiency virus infection," Clinical Infectious Diseases, vol. 35, no. 11, pp. 1414-1417, 2002.

[13] H. M. Elsheikha, "Congenital toxoplasmosis: priorities for further health promotion action," Public Health, vol. 122, no. 4, pp. 335-353, 2008.

[14] D. Nowakowska, W. Wujcicka, W. Sobala, E. Śpiewak, Z. Gaj, and J. Wilczyński, "Age-associated prevalence of Toxoplasma gondii in 8281 pregnant women in Poland between 2004 and 2012," Epidemiology and Infection, vol. 142, no. 3, pp. 656-661, 2014.

[15] H. Wilking, M. Thamm, K. Stark, T. Aebischer, and F. Seeber, "Prevalence, incidence estimations, and risk factors of Toxoplasma gondii infection in Germany: A representative, crosssectional, serological study," Scientific Reports, vol. 6, 2016.

[16] S. A. Elmore, J. L. Jones, P. A. Conrad, S. Patton, D. S. Lindsay, and J. P. Dubey, "Toxoplasma gondii: epidemiology, feline clinical aspects, and prevention," Trends in Parasitology, vol. 26, no. 4, pp. 190-196, 2010.

[17] A. L. Tian, G. X. Li, H. M. Elsheikha et al., "Seroepidemiology of Toxoplasma gondii infection in patients with liver disease in eastern China," Epidemiology and Infection, vol. 145, no. 11, pp. 2296-2302, 2017.

[18] F. Du, H. L. Feng, H. Nie et al., "Survey on the contamination of Toxoplasma gondii oocysts in the soil of public parks of Wuhan, China," Veterinary Parasitology, vol. 184, no. 2-4, pp. 141-146, 2012. 


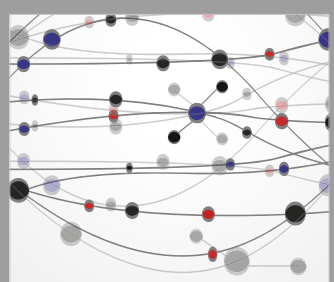

The Scientific World Journal
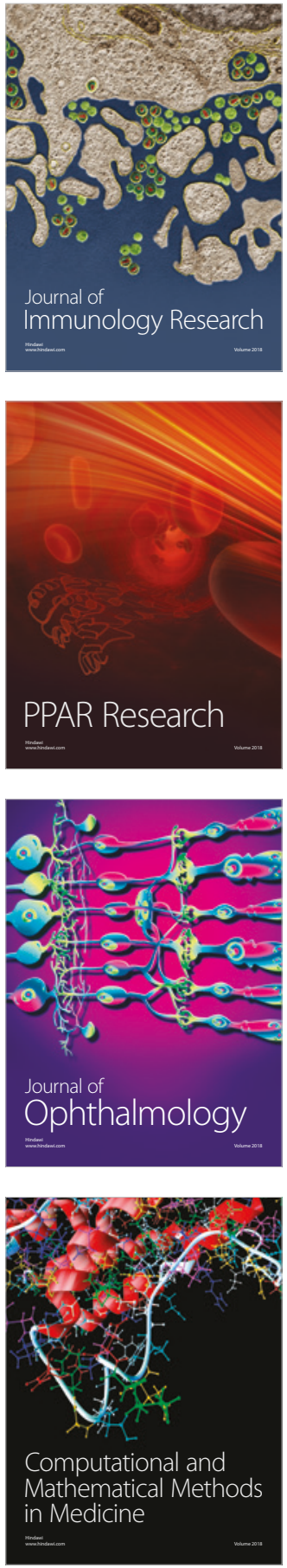

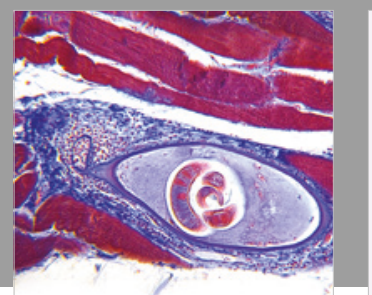

Gastroenterology Research and Practice

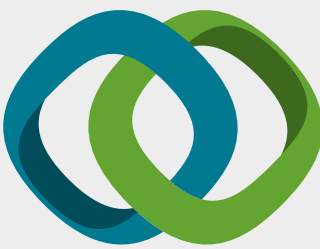

\section{Hindawi}

Submit your manuscripts at

www.hindawi.com
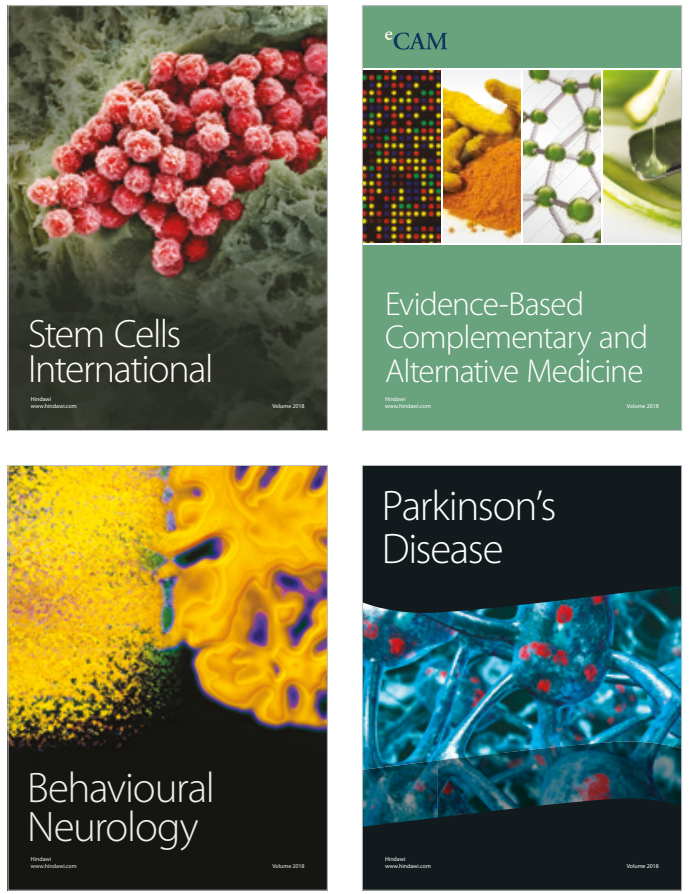

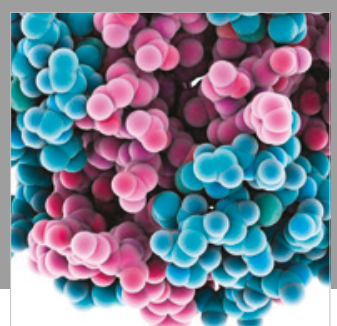

ournal of

Diabetes Research

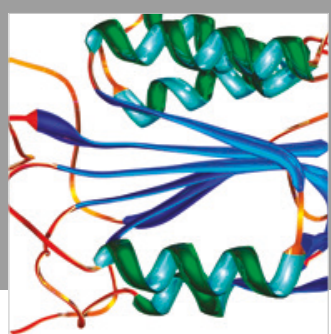

Disease Markers
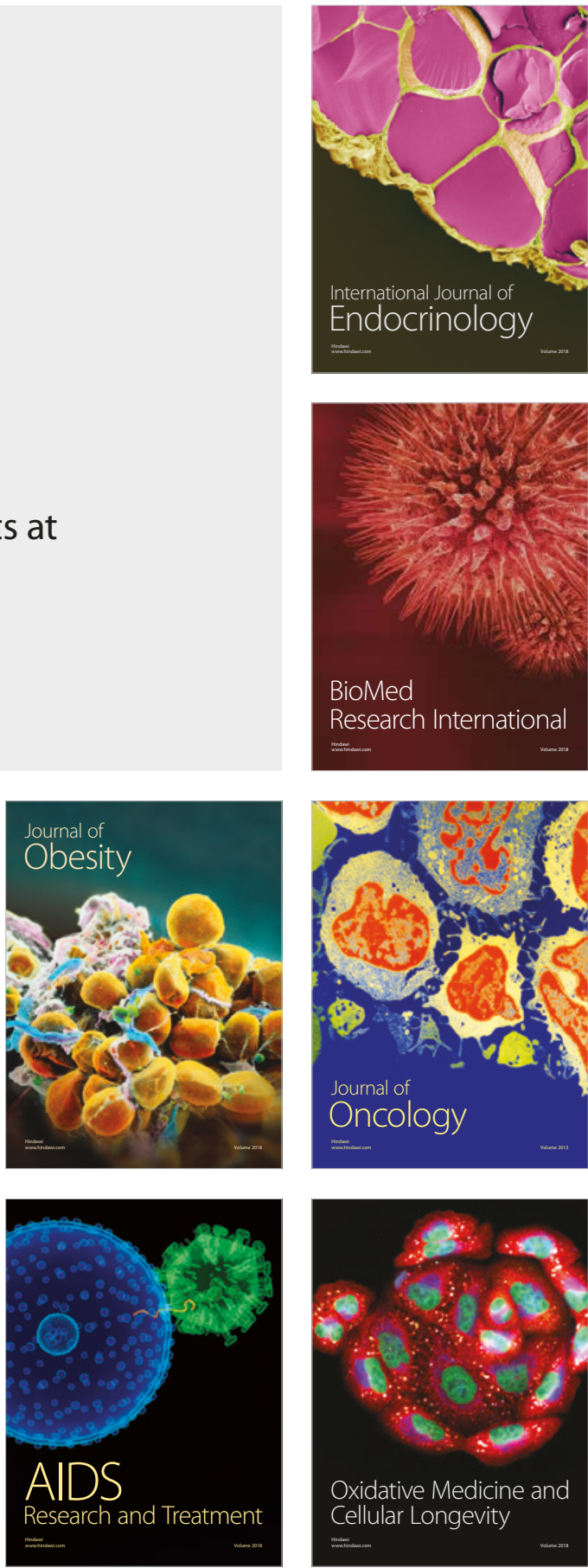